\title{
Modeling and Cross Coupling Controller Development for a 6DOF Laser Micro-Machining System
}

\author{
Serhat Kerimoglu and Melih Cakmakci*
}

\begin{abstract}
In recent years, studies on manufacturing systems have proved the importance of cooperation of positioning systems with laser cutting technology. The performance of the manufacturing system can be improved by utilizing both laser and positioning systems together. In this study, modeling and cross coupling controller development of a micromachining system which can perform on non-linear contoured surfaces is presented. Laser micromachining system is designed and assembled including a nanosecond Q-switched pulsed fiber laser, a 6-DOF hexapod manipulator, a granite table in order to absorb vibrations and an external cabin system to isolate the whole system for safety and health issues. The positioning system used here has fast response and precise positioning capabilities with a wide range of workspace. However, its performance of machining non-linear surfaces can be further improved by using a cross coupled control algorithm. On top of the loop based controllers an add-on controller is developed to improve the contouring performance by including the effects of the other feedback loops on the overall controller. Actual laser cutting results also show improvements due to the improvements in the positioning system performance.
\end{abstract}

\section{INTRODUCTION}

Over the recent years, there is increasing interest on micromachining systems for many industrial applications. Laser micromachining technology has drawn attention among other micro-manufacturing technologies due to its advantages. Laser technology features a non-contact and nearly forceless (i.e. no disturbance force) machining technique and offer many other advantages when compared to conventional manufacturing systems. Some of these can be listed as material variety, absence of machining tool dynamics, rapid process time and diversity of laser systems that eliminates size considerations in manufacturing. Some laser micromachining systems are capable of achieving down to one micron light beam cutting accuracy with sub-micrometer precision and they are able to perform on almost any material with proper settings. Popular application areas can be listed as biomedical catheter hole drilling, micro-electro-mechanical system (MEMS) fabrication applications, ink jet printer nozzle, microfluidics and production of plastic micro lens arrays [1], [2],[3], [4].

A laser micromachining system mainly consist of a laser light source which generates the laser beam, a beam delivery system that directs the laser beam to the workpiece and a positioning system to move the workpiece towards the beam. Although some systems use mirror based beam manipulation, in order to get rid of workspace limitations

Authors are with the Department of Mechanical Engineering,Bilkent University,Ankara, 06800,Turkey. email: [serhat.kerimoglu], [melihc] @bilkent.edu.tr and add some more dimensions, external positioning systems are also implemented to the overall system[5]. Many of the externally implemented positioning systems have orthogonal and translational degrees of freedom along $\mathrm{X}, \mathrm{Y}$ and $\mathrm{Z}$ axes [6]. There are some other systems which can operate on rotary axes as well [7]. Coronary stent manufacturing processes are the basic examples of such applications [8]. For more complex applications like manufacturing a 3D structure from a planar material, a laser type called excimer can be used. With an excimer laser, 3D materials can be manufactured in micrometer and nanometer scales [9]. There some other ways for 3D micro-structures fabrication like lithography and ultra precise diamond turning [10],[11].

In industry, some commercial laser micromanufacturing systems are available that can perform on nonlinear surfaces [12]. One way is to perform directly on a 3D preprocessed surface[13].

A schematic representation of a laser micromachining system is presented in Fig.1. A laser micromachining system consists of a laser system, a positioning system and control systems with a computer. For many laser systems, beam collimator(narrows the laser beam), mirror (reflects the narrowed laser beam) and focus objective (optical lens, which focuses the laser beam on a point) are combined in a single system. Precision of positioning system and spot size of the laser system are the crucial parameters of the laser micromachining systems. Quality and characteristics of machined material depends on these components.

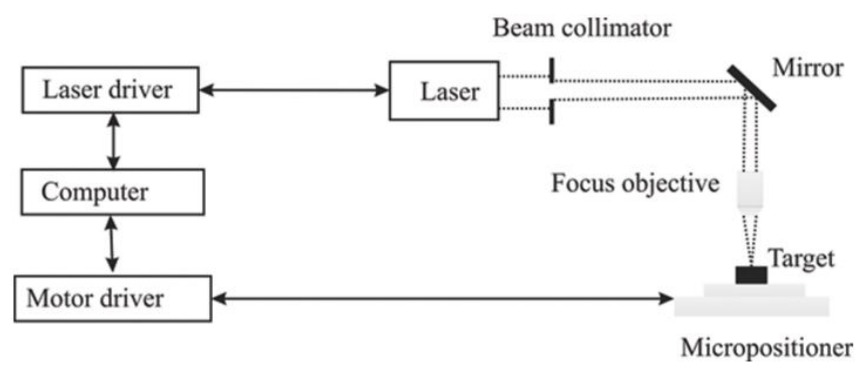

Fig. 1. Schematic Representation of a Laser MicroMachining System [14]

In order to develop a laser micromachining system that can perform on nonlinear contoured surfaces, laser and positioning systems need to be combined perfectly. In Fig. 2 , the main components of the laser micromachining system used in this work is introduced highlighting the steps of integration. 


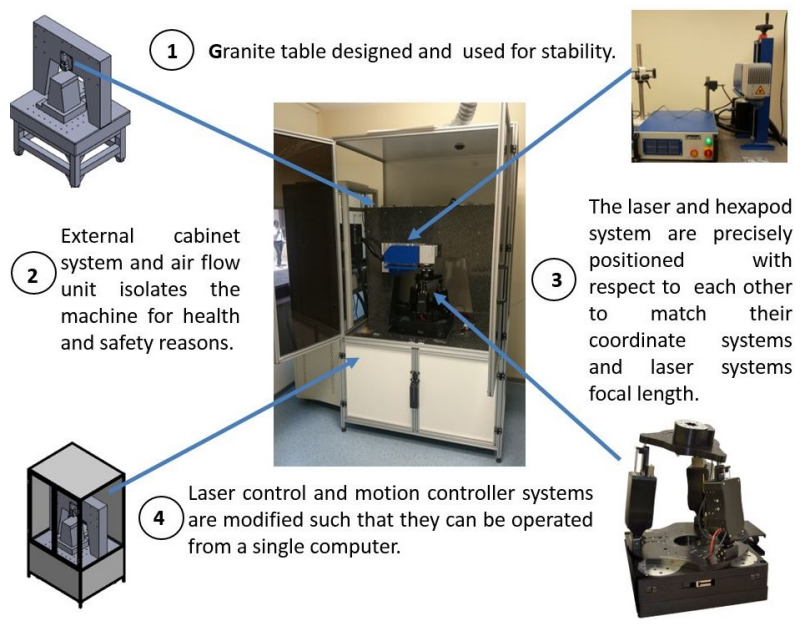

Fig. 2. Laser Micromachining System Setup

For this system a nanosecond Q-Switched Pulsed Fiber Laser and a 6-DOF hybrid hexapod positioning system from Alio Industries is used. The positioning system is a hybrid manipulator which consists of serial and parallel chains and it is composed of three parts. These parts are X-Y stage for translational motion on $\mathrm{X}$ and $\mathrm{Y}$-axes, 3-RPS tripod for translation in Z- axis and rotational motions on X and Y-axes Roll and Pitch. Remaining degree of freedom is added with a rotary actuator that enables rotation about $Z$-axis i.e. the yaw motion. Consequently, the overall positioning system has six degrees of freedom. In this machining system yaw motion is a redundant motion. However, the yaw motion actuator increases operation space of the overall system and provides a fast and continuous manufacturing.

The main motivation of this work is to develop a multiaxis laser micromachining system which is able to perform on nonlinear contoured surfaces. The selected positioning system has fast and precise positioning capabilities with a wide range of workspace. However, as will be shown in the upcoming sections its performance for machining non-linear surfaces can be further improved by using a cross coupled control algorithm on top of the loop based controllers to improve the contouring performance.

\section{MATHEMATICAL MODELS FOR THE POSITIONING SYSTEM}

The manipulator used for positioning has six degrees of freedom with a hybrid structure. A manipulation system with hybrid structure is a composition of serial and parallel kinematic chains. These structures take advantage of both serial and parallel positioning systems in one single system. The serial portion of the positioning system provides a wide work space and the parallel portion, which is a 3-RPS (RevolutePrismatic-Spherical) parallel manipulator, increases precision of the positioning system. A basic sketch of this positioning system is presented in Fig.3.

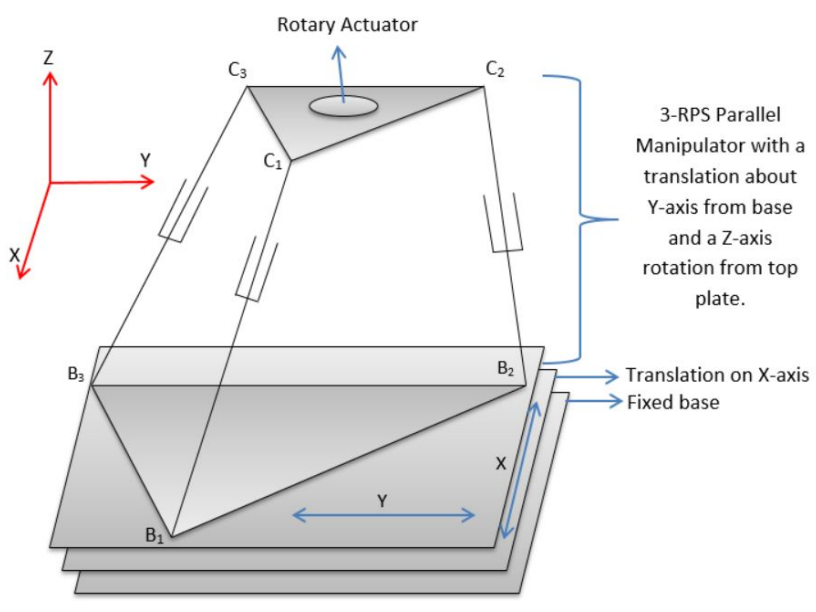

Fig. 3. Sketch of Hybrid Manipulator used as the Positioning System

In Fig. 3, $B_{i}$ and $C_{i}$ are points located at revolute and spherical joints of the 3-RPS parallel manipulator.

\section{A. Inverse Kinematics}

Position analysis of the end effector of a manipulator requires solving forward and inverse kinematics of the system. Inverse and forward kinematics analysis can be done by investigating the relationship between joints space and cartesian space of the positioning system. Solution to the inverse kinematics problem provides joint parameters of the system by using desired position of the end effector. Schematic illustration of the 3-RPS parallel manipulator is presented by Fig. 3 and Fig. 4 in perspective and as a side view respectively. Three coordinate systems are placed at some necessary points on the manipulator. These points are, at the center of the moving platform $\left(O_{1}\right)$, at the bottom of the 3-RPS $\left(O_{2}\right)$ and finally at the bottom of the whole $\operatorname{system}\left(O_{3}\right)$ respectively.

$\vec{r}$ is the position vector of the end effector of the positioning system with respect to $O_{2}$ coordinate frame. This vector also relates coordinate frames $O_{2}$ and $O_{3}$ for translational motion on $\mathrm{X}, \mathrm{Y}$ and $\mathrm{Z}$ axes. $\vec{R}$ is the position vector of the end effector of the positioning system with respect to $O_{1}$ coordinate frame and represents translational motion of overall manipulator on $\mathrm{X}, \mathrm{Y}$ and $\mathrm{Z}$ axes. $\vec{S}$ is a position vector that relates coordinate frames $\mathrm{O}_{1}$ and $\mathrm{O}_{2}$ and it represents translational motions $\mathrm{X}$ and $\mathrm{Y}$ axes of serial component of the manipulator. $\overrightarrow{q_{i}}$ relate coordinate frame $\mathrm{O}_{2}$ and $\vec{C}_{i}$ and they are position vectors of spherical joints on the moving platform. $h_{1}, h_{2}$ and $h_{3}$ are the constant offsets on Zaxis between linear actuators at the bottom of the positioning system. $\vec{r}, \vec{R}$ and $\vec{S}$ vectors can be presented respectively as shown in (1).

$$
\begin{gathered}
\vec{r}=\left(\begin{array}{lll}
r_{x} & r_{y} & r_{z}
\end{array}\right)^{T}, \\
\vec{R}=\left(\begin{array}{lll}
R_{x} & R_{y} & R_{z}
\end{array}\right)^{T} \\
\vec{S}=\left(\begin{array}{lll}
S_{x} & S_{y} & S_{z}
\end{array}\right)^{T}
\end{gathered}
$$




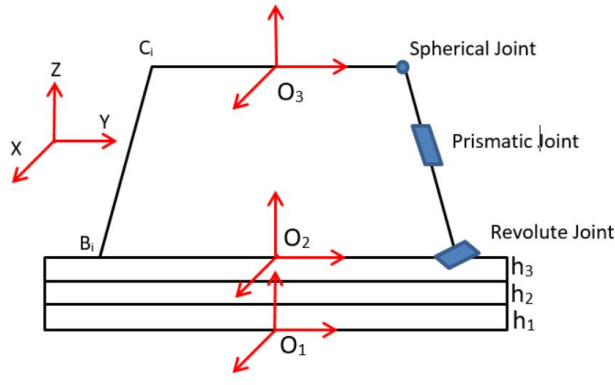

Fig. 4. Sketch of the Manipulator in Case of Coordinate Systems

Position of the spherical joints with respect to the $\mathrm{O}_{3}$ coordinate frame and positions of the revolute joints with respect to the $\mathrm{O}_{2}$ coordinate frame are presented respectively $\overrightarrow{C_{1}}, \overrightarrow{C_{2}}, \overrightarrow{C_{3}}$ and $\overrightarrow{B_{1}}, \overrightarrow{B_{2}}$ and $\overrightarrow{B_{3}}$ vectors.

For the transformation calculations between $\mathrm{O}_{2}$ and $\mathrm{O}_{3}$ coordinate frames of the 3-RPS manipulator, a rotation matrix $\left(R_{B C}\right)$ is used. Due to the geometrical properties of the positioning system the Euler Sequence of the transformation is chosen as [3,1,2] based on [15] as shown in (2).

$$
R_{B C}=\left[\begin{array}{lll}
u_{x} & v_{x} & w_{x} \\
u_{y} & v_{y} & w_{y} \\
u_{z} & v_{z} & w_{z}
\end{array}\right]
$$

where $\mathrm{u}, \mathrm{v}$ and $\mathrm{w}$ are direction cosines, and then the resulting $R_{B C}$ is given in (3).

$$
R_{B C}=
$$

$\left[\begin{array}{ccc}\mathrm{c}(\gamma) \mathrm{c}(\alpha)+\mathrm{s}(\alpha) \mathrm{s}(\beta) \mathrm{s}(\gamma) & -\mathrm{c}(\gamma) \mathrm{s}(\alpha)+\mathrm{c}(\alpha) \mathrm{s}(\beta) \mathrm{s}(\gamma) & \mathrm{c}(\beta) \mathrm{s}(\gamma) \\ \mathrm{c}(\beta) \mathrm{s}(\alpha) & \mathrm{c}(\beta) \mathrm{c}(\alpha) & -\mathrm{s}(\beta) \\ -\mathrm{s}(\gamma) \mathrm{c}(\alpha)+\mathrm{c}(\gamma) \mathrm{s}(\beta) \mathrm{s}(\alpha) & \mathrm{s}(\gamma) \mathrm{s}(\alpha)+\mathrm{c}(\alpha) \mathrm{s}(\beta) \mathrm{c}(\gamma) & \mathrm{c}(\beta) \mathrm{c}(\gamma)\end{array}\right]$

In $R_{B C}, \alpha, \beta$ and $\gamma$ are Euler angles and $\mathrm{s}(\alpha)=\sin (\alpha)$ and $\mathrm{c}(\alpha)=\cos (\alpha)$. Scalar values for limb lengths and $\overrightarrow{q_{i}}$ vectors for the system can be derived using (4) and (5).

$$
\begin{gathered}
\overrightarrow{q_{i}}=R_{B C} \mathrm{O}_{3} C_{i} \\
L_{i}=\left\|\vec{r}+\overrightarrow{q_{i}}-\overrightarrow{B_{i}}\right\|
\end{gathered}
$$

where $\overrightarrow{\mathrm{O}_{3} B_{i}}$ and $\overrightarrow{\mathrm{O}_{3} C_{i}}$ are representation of $\overrightarrow{B_{i}}$ and $\vec{C}_{i}$ vectors with respect to $\mathrm{O}_{3}$ coordinate frame.

\section{B. Forward Kinematics}

Forward kinematics solution of a positioning system provides position of the end effector of the system by using joint parameters. For the specified 3-RPS parallel manipulator, limb angle $\varphi_{i}$ and limb length $L_{i}$ are joint parameters.

$$
\begin{gathered}
\overrightarrow{l_{1}}=\left(\begin{array}{lll}
\mathrm{c}\left(\varphi_{1}\right) & 0 & \mathrm{~s}\left(\varphi_{1}\right)
\end{array}\right)^{T} \\
\overrightarrow{l_{2}}=\left(\begin{array}{lll}
-\mathrm{c}\left(\varphi_{2}\right) / 2 & \mathrm{c}\left(\varphi_{2}\right) \sqrt{3} / 2 & \mathrm{~s}\left(\varphi_{2}\right)
\end{array}\right)^{T} \\
\overrightarrow{l_{3}}=\left(\begin{array}{lll}
\mathrm{c}\left(\varphi_{1}\right) & -\mathrm{c}\left(\varphi_{2}\right) \sqrt{3} / 2 & -\mathrm{s}\left(\varphi_{2}\right)
\end{array}\right)^{T} \\
\overrightarrow{L_{1}}=\overrightarrow{l_{1}} L_{1}, \overrightarrow{L_{2}}=\overrightarrow{l_{2}} L_{2}, \overrightarrow{L_{3}}=\overrightarrow{l_{3}} L_{3}
\end{gathered}
$$

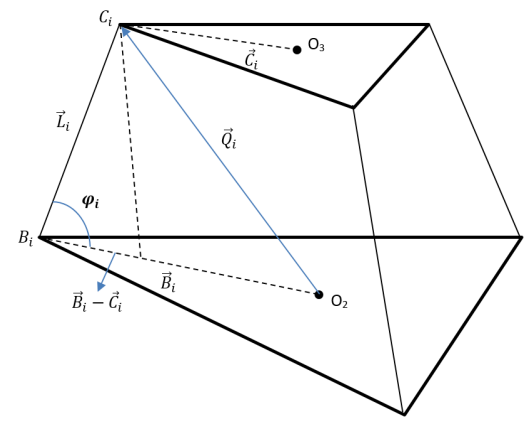

Fig. 5. Position Analysis for Spherical Joints

$\overrightarrow{l_{1}}, \overrightarrow{l_{2}}$ and $\overrightarrow{l_{3}}$ are the unit vectors and $\overrightarrow{L_{1}}, \overrightarrow{L_{2}}$ and $\overrightarrow{L_{3}}$ are vectors for the legs. Then, (10) can be used to find the positions of spherical joint coordinates with respect to $\mathrm{O}_{2}$ coordinate system. These vectorial relationships are illustrated on Fig.5.

$$
\overrightarrow{O_{2} C_{i}}=\overrightarrow{O_{2} B_{i}}-L_{i} c\left(\varphi_{i}\right)+L_{i} s\left(\varphi_{i}\right)
$$

It is important to notice that, $\mathrm{O}_{2} \vec{C}_{i}$ is the representation of $\overrightarrow{q_{i}}$ with joint parameters $\varphi_{i}$ 's and $L_{i}$ 's instead of cartesian space parameters. They are the position vectors between $\mathrm{O}_{2}$ coordinate frame and the spherical joints. In this paper $\overrightarrow{Q_{i}}$ is used instead of $\vec{O}_{2} C_{i}$ for simplification of future calculations. Consequently, we can use (11) to find the Cartesian coordinates of the end effector.

$$
\overrightarrow{Q_{c}}=\frac{\overrightarrow{Q_{1}}+\overrightarrow{Q_{2}}+\overrightarrow{Q_{3}}}{3}
$$

Nonlinear equations are given in Eqn. (12) to (14) are the relations between limb lengths and angles $\varphi_{i}$ 's. By solving these equations, $\varphi_{i}$ 's can be obtained numerically and used as inputs of forward kinematics equations.

$$
\begin{aligned}
& f_{1}=\left(\frac{L_{1}}{d_{b}}\right)^{2}+\left(\frac{L_{2}}{d_{b}}\right)^{2}+3-3\left(\frac{d_{c}}{d_{b}}\right)^{2}+\left(\frac{L_{1}}{d_{b}}\right)\left(\frac{L_{2}}{d_{b}}\right) c\left(\varphi_{1}\right) c\left(\varphi_{2}\right) \\
& -2\left(\frac{L_{1}}{d_{b}}\right)\left(\frac{L_{2}}{d_{b}}\right) s\left(\varphi_{1}\right) s\left(\varphi_{2}\right)-3\left(\frac{L_{1}}{d_{b}}\right) c\left(\varphi_{1}\right)-3\left(\frac{L_{2}}{d_{b}}\right) c\left(\varphi_{2}\right)=0 \\
& f_{2}=\left(\frac{L_{2}}{d_{b}}\right)^{2}+\left(\frac{L_{3}}{d_{b}}\right)^{2}+3-3\left(\frac{d_{c}}{d_{b}}\right)^{2}+\left(\frac{L_{2}}{d_{b}}\right)\left(\frac{L_{3}}{d_{b}}\right) c\left(\varphi_{2}\right) c\left(\varphi_{3}\right)- \\
& 2\left(\frac{L_{2}}{d_{b}}\right)\left(\frac{L_{3}}{d_{b}}\right) s\left(\varphi_{2}\right) s\left(\varphi_{3}\right)-3\left(\frac{L_{2}}{d_{b}}\right) c\left(\varphi_{2}\right)-3\left(\frac{L_{3}}{d_{b}}\right) c\left(\varphi_{3}\right)=0 \\
& f_{3}=\left(\frac{L_{1}}{d_{b}}\right)^{2}+\left(\frac{L_{3}}{d_{b}}\right)^{2}+3-3\left(\frac{d_{c}}{d_{b}}\right)^{2}+\left(\frac{L_{1}}{d_{b}}\right)\left(\frac{L_{3}}{d_{b}}\right) c\left(\varphi_{1}\right) c\left(\varphi_{3}\right)- \\
& 2\left(\frac{L_{1}}{d_{b}}\right)\left(\frac{L_{3}}{d_{b}}\right) s\left(\varphi_{1}\right) s\left(\varphi_{3}\right)-3\left(\frac{L_{1}}{d_{b}}\right) c\left(\varphi_{1}\right)-3\left(\frac{L_{3}}{d_{b}}\right) c\left(\varphi_{3}\right)=0
\end{aligned}
$$

By computing $\varphi_{i}$ 's and $\overrightarrow{L_{i}}$ 's from the kinematic analysis, we can now obtain rotational position of the upper platform of 3-RPS parallel manipulator.

$$
\begin{aligned}
\vec{u} & =\left(\begin{array}{lll}
u_{x} & u_{y} & u_{z}
\end{array}\right)^{T} \\
\vec{v} & =\left(\begin{array}{lll}
v_{x} & v_{y} & v_{z}
\end{array}\right)^{T}
\end{aligned}
$$




$$
\begin{gathered}
\vec{w}=\left(\begin{array}{lll}
w_{x} & w_{y} & w_{z}
\end{array}\right)^{T} \\
\vec{u}=\left(\overrightarrow{Q_{1}}-\overrightarrow{Q_{c}}\right) / \overrightarrow{C_{1}}(1) \\
\vec{v}=\left(\overrightarrow{Q_{2}}-\overrightarrow{Q_{3}}\right) /\left(\overrightarrow{C_{2}}(2)-\overrightarrow{C_{3}}(2)\right) \\
\vec{w}=\vec{u} \times \vec{v}
\end{gathered}
$$

Solutions of Euler angles can be obtained as shown in (21)-(23),

$$
\begin{gathered}
\alpha=\arctan \left(\overrightarrow{w_{x}} / \overrightarrow{w_{z}}\right) \\
\beta=\arctan \left(\overrightarrow{u_{y}} / \overrightarrow{v_{y}}\right) \\
\gamma=\arcsin \left(-\overrightarrow{w_{y}}\right)
\end{gathered}
$$

$\mathrm{X}, \mathrm{Y}$ and $\mathrm{Z}$ axis translational motions can bu computed by using (11),

$$
\begin{aligned}
X & =Q_{c}(1) \\
Y & =Q_{c}(2) \\
Z & =Q_{c}(3)
\end{aligned}
$$

So far, the position analysis of 3 - RPS parallel part of the positioning system is presented. The kinematic solutions of remaining serial components of the overall positioning system can be added directly to the kinematics of 3-RPS manipulator. Positioning system in this work have three more actuation systems for remaining motions which are translational motions in $\mathrm{X}$ and $\mathrm{Y}$ axes and a rotational motion about $\mathrm{Z}$ axis $(\gamma) . P_{x}$ and $P_{y}$ are defined for actuator positions on $\mathrm{X}$ and $\mathrm{Y}$ axes and use $\gamma_{a}$ to define actuator position for rotational motion about $\mathrm{Z}$ axis. Finally, remaining three degrees of freedom can be obtained by using following relations.

$$
\begin{gathered}
X=P_{x}+Q_{c}(1) \\
Y=P_{y}+Q_{c}(2) \\
\gamma=\gamma_{a}
\end{gathered}
$$

C. Dynamic Model of Positioning System with SimMechanics

MATLAB/SimMechanics simulation software enables to dynamically model three dimensional mechanical systems. In this study, for simulation and control development, a SimMechanics model is created for the positioning of laser the micromachining system using couple of steps. A CAD model of the positioning system is established in SolidWorks and imported to SimMechanics to obtain a dynamic model and it is created by using the structural properties and kinematic models of the positioning system.

The input output relationships for the inverse kinematics, forward kinematics and SimMechanics model is given in Fig. $6 \mathrm{a}$ and Fig. $6 \mathrm{~b}$ based on the mathematical relationships developed in this section. As shown in part (b), the inputs to the SimMechanics model is assumed to be the actuator force commands that will be given from the controllers. This model can be used to simulate the overall behavior of the original system and the cross coupling controller developed in the next section. (a)

(b)
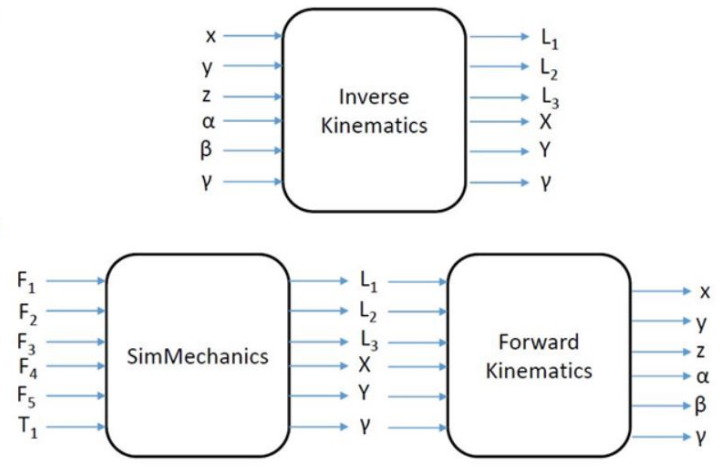

Fig. 6. Representative Schema of the Manipulator Model

\section{CROSS-COUPLED CONTROLLER DEVELOPMENT}

Positioning system uses error based feedback controllers such as PID controllers using the actuator position error in the system. Due to the complex nature of the design process the baseline controllers provided for this system featured a single loop based controllers that are operated individually as shown in Fig. 7. This structure may be suitable for some basic manipulation, however for profiles that span in 3D with non-linear trajectories the performance of the system may suffer especially in high speeds.

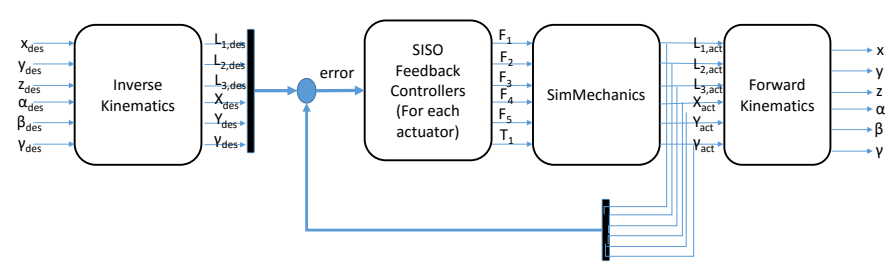

Fig. 7. Loop Based Controller Block Diagram for the Manipulator System

The desired trajectory shown in Fig. 8 presents such a low performance case. The trajectory was uploaded to the software of the positioning system with the original controller and tuned. Then the measured and desired actuator position information are gathered from the encoders of the actuators of the positioning system are recorded simultaneously. By using the forward kinematics of the positioning system, measured actuator informations are converted to measured trajectory tracking informations. As it can be seen from this figure the performance of the system suffers especially in the short range quick movement spanned in xyz directions. Outcome of the observations on the performance of the actuators and comparison of the measured and desired trajectory tracking performances show that, the performance of the positioning system decreases due to the position tracking performance of the limb actuators. 


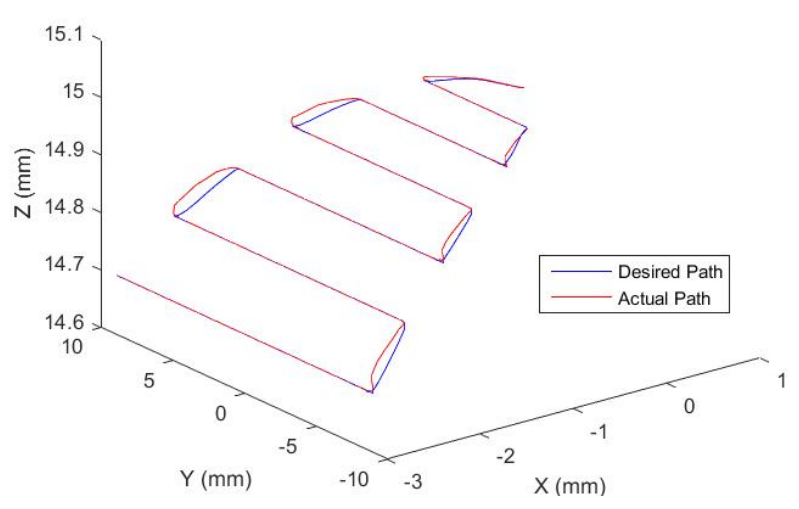

Fig. 8. Trajectory Tracking Plot of Measured vs Desired Position

One way to the remedy the contouring error seen in Fig. 8 is to use a MIMO controller structure that uses the information and action from multiple loops simultaneously. While designing a MIMO controller is more complicated than designing a single loop based SISO controller, some techniques exist to approach the design procedure more in a more structured way. Using a cross coupled controller (CCC) is one of them.

\section{A. Contour Error and Cross Coupling}

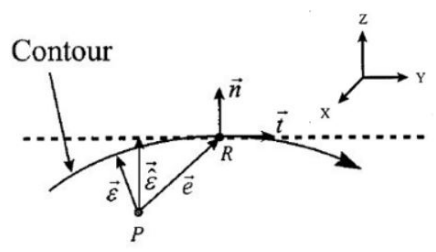

Fig. 9. Geometrical Relations of Contour Error (adopted from[16])

Cross-coupled control is a special type of multi-inputmulti-output (MIMO) control, which uses the contour error of the positioning system. A typical block diagram for a cross coupled controller is given in references like [17] and also in Fig.10 in this paper. In this block diagram, $C_{x}$ and $C_{y}, C_{z}$ are the coupling gains whereas $\varepsilon, e_{x}, e_{y}, e_{z}$ are the contour error, $\mathrm{x}$-axis tracking error, $\mathrm{y}$-axis tracking error and $\mathrm{z}$-axis tracking error, respectively. The contour error, $\varepsilon$, is obtained using (30).

$$
\varepsilon=C_{x} e_{x}+C_{y} e_{y}+C_{z} e_{z}
$$

Although CCC is first introduced with constant gains, the term CCC is generally used for CCC with variable coupling gains (i.e. $C_{x}$ and $C_{y}$ ) as proposed in [18]. For a nonlinear contour, calculation of these gains is can be complicated. A contour error approximations can be used to simplify the coupling gain computation. The contour error vector approach can be explained using the geometrical relationship is given in Fig. 9. In this figure, $\vec{t}$ and $\vec{n}$ are the normalized tangential and normal vectors respectively based on the actual position, $P$ and the reference position, $R$. The contouring error, $\vec{\varepsilon}$, is defined as the vector from the actual position to the nearest point on the line that

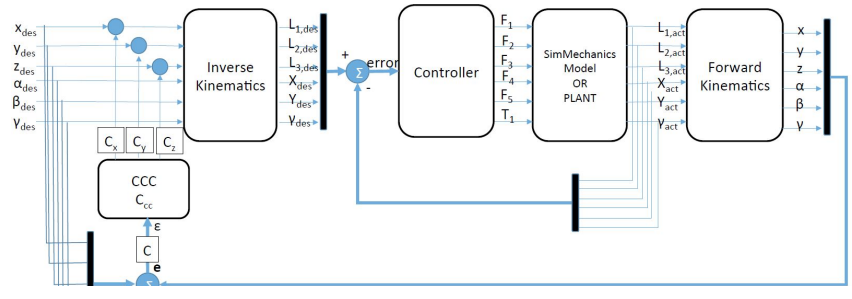

Fig. 10. Developed Controller with the CCC controller structure

passes through the reference position tangentially in the direction of $\vec{t}$. The estimated tracking error, $\vec{\varepsilon}$, is equal to $\langle\vec{e}, \vec{n}\rangle$ where $\vec{e}$ is the tracking error and $\langle.,$.$\rangle is the$ inner product operator. The contour error is calculated as $|\vec{\varepsilon}|=\sum_{i} C_{i} e_{i}(i=x, y, z)$ where $C_{i}$ is coupling gain and $e_{i}$ is the corresponding tracking error for each axis. By equating the two representations of estimated contour error vector, $\vec{\varepsilon}$, cross coupling gains $\left(C_{x}, C_{y}, C_{z}\right)$ are found as $C_{i}=n_{i}(i=x, y, z$,$) . In other words, cross coupling gains$ at a specific point on the contour are the elements of $\vec{n}$ of the contour at that point. A first order transfer function with tunable gain and time delay parameters is used for the cross coupled controller, $C_{c c}$, for a fast responding, low-cost and robust coupling effect. The advantages and stability study for a 3DOF positioning system was given in [17]. In essence, the approach proposed here is stable when the feedback controller is stable and its implementation is computationally effective.

\section{B. Implementation and Simulations}

The cross coupled controller discussed in the previous section can be implemented on the system to improve its contouring performance. Fig. 10 illustrates the actual implementation of this controller as an add-on to the loop based controller given in Fig. 7. It is important to note that the rotational angles does not contribute to the $\mathrm{CCC}$ calculations in the system. This is done by simply ignoring these signals for the block diagram for graphical simplicity. However, this can also be formally done by assuming $C_{\alpha}, C_{\beta}, C_{\gamma}$ as zeros in calculations.

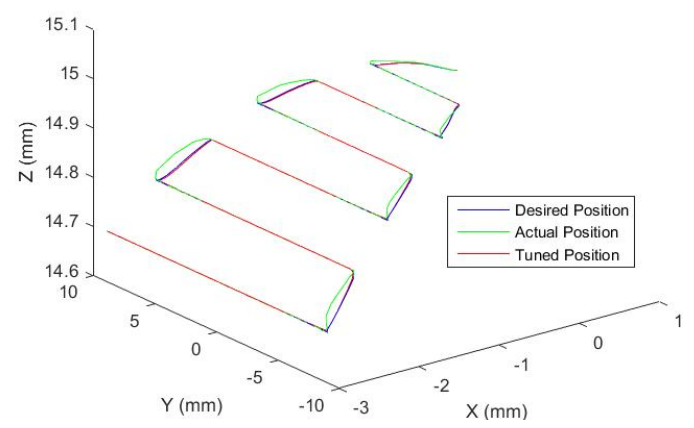

Fig. 11. Tuned Trajectory Comparison with Desired and Measured Results (Simulation)

In order to observe the improvements of the overall laser 
micromachining system for nonlinear contoured surfaces, the same experiment is performed with the cross coupled controller active with the tuned controllers. Desired nonlinear trajectory is uploaded to the positioning system and the performance of the laser micromachining system on a cylindrical surface is observed. Fig. 11 shows the results of performed experiment. The improved performance of the controller tracking with the new controller is seen clearly with much less error in tracking. Table 1 presents the cumulative tracking error of the actuators and projection of the error across the whole trajectory. As it can be seen from the results the final system gives a vastly improved accuracy in positioning under a non-linear 3D trajectory.

TABLE I

Root Mean Square of Position Errors for Trajectory TRACKING BEFORE AND AFTER THE NEW CONTROLLER IS IMPLEMENTED

\begin{tabular}{ccc}
\hline \hline & Original Cont. Error [mm] & New Cont. Error[mm] \\
\hline \hline L1 & 4.15 & 0.5 \\
L2 & 2 & 0.23 \\
L3 & 2.36 & 0.32 \\
\hline $\mathrm{X}$ & 1.3 & 0.22 \\
$\mathrm{Y}$ & 1.8 & 0.3 \\
$\mathrm{Z}$ & 1.84 & 0.47 \\
\hline
\end{tabular}

\section{CONCLUSIONS}

In this paper a control algorithm for a multi-axis laser micromachining system which is able to perform on nonlinear contoured surfaces is presented. Even though the original positioning system has fast and precise positioning capabilities with a wide range of workspace, its performance of machining non-linear surfaces can be further improved by using a cross coupled control algorithm. On top of the loop based controllers an add-on controller is used to improve the contouring performance by including the effects of the other feedback loops on the overall controller. Fig. 12 and Fig. 13 show the actual laser machining results obtained with the original and improved controller respectively. The performance improvement of the new controller based on the actuator interactions is clearly seen from the end product. The integration of the laser controller and obtaining a more comprehensive MIMO controller based on dynamic modeling are the future work on this topic.

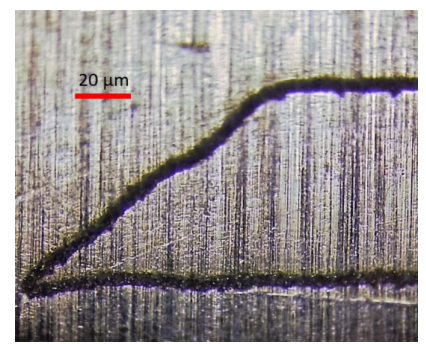

Fig. 12. Laser Micromachining Result with Controller

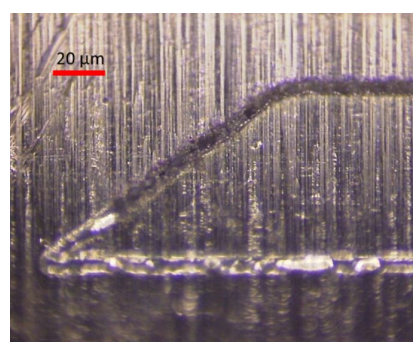

Fig. 13. Laser Micromachining Result with the New Controller

\section{ACKNOWLEDGEMENT}

Authors would like to thank Dr. Yigit Karpat, Dr. Uluc Saranli and Dr. Sakir Baytaroglu for their valuable advice and technical guidance.

\section{REFERENCES}

[1] M. Gower, "Industrial applications of laser micro-machining," Optics Express, vol. 7, no. 2, pp. 56-67, 2000.

[2] A. G. J.P.H. Burt and J. T. C.J. Hayden, "Laser micromachining of biofactory-on-a-chip devices," Proc. SPIE, vol. 4637, pp. 305-317, 2002.

[3] I. S.-B. ForK.L. Boehlen, "Laser micromachining of high-density optical structures on large substrates," Proc. SPIE, vol. 5339, pp. 118126, 2004.

[4] A. S. Holmes, J. E. Pedder, and K. L. Boehlen, "Advanced laser micromachining processes for mems and optical applications," Proc. SPIE, vol. 6261, 2006.

[5] I. S. K. Edin Golubovic, E. A. B. Ahmet O. Nergiz, and A. Sabanovic, "Design and control of laser micromachining workstation," IEEE International Workshop on Advanced Motion Control, vol. 12, 2012.

[6] D. B. Pavel Penchev, S. Dimov, and S. L. Soo, "Novel manufacturing platform for scale up production of miniaturized parts," INTERNATIONAL WORKSHOP ON MICROFACTORIES, pp. 231 - 238, 2014.

[7] AEROTECH, "Capabilities in laser processing and micromachining," Aug. 2016.

[8] B. P. Ali Gkhan Demir and C. A. Biffi, "Fibre laser cutting and chemical etching of az31 for manufacturing biodegradable stents," Hindawi Publishing Corporation Advances in Materials Science and Engineering, vol. 2013, 2013.

[9] Q. Xia and S. Y. Chou, "Applications of excimer laser in nanofabrication," Applied Physics, vol. 98, pp. 9-59, 2010.

[10] E. Türeyen, Y. Karpat, and M. Çakmakcı, "Development of an iterative learning controller for polymer based micro-stereolithography prototyping systems," in 2016 American Control Conference (ACC), pp. 852-857, IEEE, 2016.

[11] L. Li and A. Y. Yi, "Microfabrication on a curved surface using 3d microlens array projection," Journal of Micromechanics and Microengineering, vol. 19, 2009.

[12] ALPHANOV, "Development and design of custom laser micromachining systems," Aug. 2016.

[13] R. R. H. Pantsar and A. J. P. Laakso, "Advances in 3d laser processing in mold technology," 25th International Congress on Applications of Lasers and Electro Optics, 2006.

[14] M. L. A. Cinthya Toro Salazar and C. A. Rinaldi, "Nanostructuring of material surfaces by laser ablation,” InTech, vol. 10.5772/62638, 2016.

[15] J. Diebel, "Representing attitude: Euler angles, unit quaternions, and rotation vectors," Matrix, vol. 58, no. 15-16, pp. 1-35, 2006.

[16] S.-S. Yeh and P.-L. Hsu, "Estimation of the contouring error vector for the cross-coupled control design," IEEE/ASME transactions on mechatronics, vol. 7, no. 1, pp. 44-51, 2002.

[17] N. G. Ulu, E. Ulu, and M. Cakmakci, "Design and analysis of a modular learning based cross-coupled control algorithm for multiaxis precision positioning systems," International Journal of Control, Automation and Systems, vol. 14, no. 1, pp. 272-281, 2016.

[18] Y. Koren and C.-C. Lo, "Variable-gain cross-coupling controller for contouring," CIRP Annals-Manufacturing Technology, vol. 40, no. 1, pp. 371-374, 1991. 